\title{
Exclusion of the genes CDKN2 and PTEN as causative gene defects in Li-Fraumeni syndrome
}

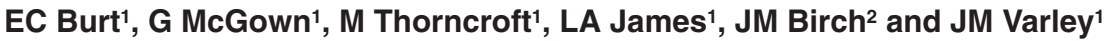 \\ ${ }^{1}$ CRC Section of Molecular Genetics, Paterson Institute for Cancer Research, Wilmslow Road, Manchester M20 4BX, UK; ${ }^{2} \mathrm{CRC}$ Paediatric and Familial Cancer \\ Research Group, Royal Manchester Children's Hospital, Manchester M27 1HA, UK
}

Summary We have analysed Li-Fraumeni syndrome families, previously shown to be negative for mutations in TP53, for mutations to the tumour suppressor genes PTEN and CDKN2. These genes function in cell cycle progression or are mutated in a variety of tumours. We have detected no mutations in the family members tested.

Keywords: Li-Fraumeni syndrome; PTEN; CDKN2; p16; p19ARF

Li-Fraumeni syndrome (LFS) is an autosomal, dominant, inherited cancer disorder, characterized by a broad but specific spectrum of tumours observed at an early age, in particular a predominance of bone and soft tissue sarcomas, brain and breast cancer ( $\mathrm{Li}$ et al, 1988). LFS is defined by a strict clinical definition, described previously ( $\mathrm{Li}$ et al, 1988). This definition has been relaxed to include Li-Fraumeni-like (LFL) cases (Birch et al, 1994).

In 1990, the tumour suppressor gene TP53 was identified as the candidate for mutation in a number of LFS families (Malkin et al, 1990). To date more than 50 families have been identified with germline TP53 mutations (Varley et al, 1997a). Previously we have detected mutations in approximately $70 \%$ of LFS and $20 \%$ of LFL families, when all exons, including non-coding regions, have been sequenced (Varley et al, 1997b). Therefore, 30\% of LFS and the majority of LFL families do not have a detectable germline mutation within the coding region of TP53. The p53 protein functions in checkpoint control at G1/S and G2 phase of the cell cycle. It is feasible that characterization of related genes, for example other cell cycle inhibitors, may identify germline mutations within these families. We have selected genes for analysis that are involved in p53-related pathways or are known to be mutated in Li-Fraumeni-associated tumours, in particular the tumour suppressor genes $C D K N 2$, which encodes p16 and p19ARF, and PTEN. Like p53, both p16 and p19ARF function as negative regulators of the cell cycle, with the 16 gene shown to be mutated in many tumour types (Kamb, 1995; Quelle et al, 1995). PTEN mutations have also been observed in a number of tumours (Steck et al, 1997). We have analysed 16 families, previously shown to be negative for mutations to TP53 (Varley et al, 1997b), for mutations to these sequences.
Received 7 August 1998

Revised 28 October 1998

Accepted 29 October 1998

Correspondence to: JM Varley

\section{MATERIALS AND METHODS}

Families were ascertained as previously described (Varley et al, 1997b). Blood samples were obtained from either the proband or a first-degree relative in each family (Table 1). DNA was isolated from whole blood using the Dynabeads DNA direct System (Dynal), according to manufacturers instructions.

Genomic DNAs were amplified by nested polymerase chain reaction (PCR) to generate products corresponding to each exon of $P T E N$ and CDKN2. Primers and PCR conditions are available from authors. PCR products were sequenced directly using either an ABI377 Sequencer with Dye Primer chemistry (Perkin-Elmer Applied Biosystems), or a Sequenase kit (USB).

Table 1 Mutation analysis of PTEN and CDKN2 in LFS and LFL families ${ }^{a}$

\begin{tabular}{ccll}
\hline Family $^{\mathrm{b}}$ & Person $^{\mathrm{b}}$ & \multicolumn{2}{c}{ Mutation found $^{\mathrm{c}}$} \\
\cline { 3 - 4 } & & Nucleotide change $^{\mathrm{d}}$ & \multicolumn{1}{c}{ Amino acid change $^{-}$} \\
LFS Families & & & \\
22 & III-5 & None & \\
81 & III-5 & None & \\
82 & IV-5 & None & \\
88 & II-2 & None & \\
119 & III-1 & None & \\
1779 & III-7 & None & \\
LFL Families & & & \\
80 & V-4 & None & \\
253 & IV-2 & CDKN2 : 442 G>A & p16: Alal48Thre \\
328 & II-3 & None & \\
338 & III-2 & None & \\
348 & III-2 & None & \\
352 & IV-1 & None & \\
353 & III-3 & None & \\
2093 & IV-3 & None & \\
2613 & III-1 & None & \\
2634 & III-3 & None & \\
& & & \\
\hline
\end{tabular}

aFor details of tumour types and relationship to proband in each family, refer to Varley et al (1997b). ${ }^{\text {PFamily }}$ and Person numbers are as previously (Varley et al, 1997b). Includes mutations found in PTEN and CDKN2. dNucleotide positions are derived from the amended p16 gene sequence (Okamoto et al, 1994). eAla148Thr is a common polymorphism, previously reported as Ala140Thr (Hussussian et al, 1994). 


\section{RESULTS AND DISCUSSION}

We report the results of sequencing six LFS and ten LFL samples for mutations in tumour suppressor genes PTEN and CDKN2. The $C D K N 2$ gene consists of four exons $(1 \alpha, 1 \beta, 2$ and 3$)$, and encodes two proteins: 16 and p19ARF. The $\mathrm{p} 16$ protein is encoded by exons $1 \alpha, 2$ and 3, and functions as a negative regulator in the G1 phase of the cell cycle. The pl6 gene is a major target in carcinogenesis, and has been shown to be inactivated in a number of tumour cell lines and primary tumours including adenocarcinomas and glioblastoma (Kamb, 1995). Germline mutations in the gene can result in hereditary predisposition to the development of melanoma and pancreatic cancer (Hussussian et al, 1994; Foulkes et al, 1997). In addition, an alternatively spliced transcript, encoding p19ARF, is derived from the same locus as p16 and contains a novel first exon (1 $\beta)$, located upstream of exon $1 \alpha$. Both transcripts share exons 2 and 3 but are translated in a different reading frame, and hence p16 and p19ARF have no amino acid similarity. The p19 ${ }^{\mathrm{ARF}}$ protein also functions to block cell cycle progression at G1 and G2, although in a p53-dependent manner (Quelle et al, 1995). We have been unable to identify any germline mutations in either the p16 or p19ARF genes in the Li-Fraumeni families tested, although we have detected a single heterozygous polymorphism at codon 148 of p16 in IV-2, family 253 (Table 1). This polymorphism is prevalent in the population (Hussussian et al, 1994), and has no effect on p19 ${ }^{\text {ARF }}$.

The tumour suppressor gene PTEN (MMAC1) was isolated by mapping of homozygous deletions commonly found in glioblastomas (Li et al, 1997). Subsequently somatic mutations in PTEN have been observed in a number of tumours including breast and brain tumours, and malignant melanoma (Steck et al, 1997). Germline mutations in the gene have been identified in Cowden syndrome, which is associated with early onset breast cancer (Tsou et al, 1997). However, a recent study of 136 breast cancer families has shown that PTEN is not linked to familial breast cancer (Chen et al, 1998). The gene encodes a dual specificity phosphatase, with enzymatic activity required for tumour suppressor function (Myers et al, 1997). We have not detected any PTEN mutations in the families analysed.

We have therefore excluded these genes as candidates for mutation in Li-Fraumeni syndrome. However, this does not preclude other cell cycle control genes as targets for mutation, for example the $\mathrm{Rb}$ and $\mathrm{p} 21$ genes.

\section{REFERENCES}

Birch JM, Hartley AL, Tricker KJ, Prosser J, Condie A, Kelsey AM, Harris M, Morris Jones PH, Binchy A, Crowther D, Craft AW, Eden OB, Evans DGR, Thompson E, Mann JR, Martin J, Mitchell ELD and Santibanez-Koref MF (1994) Prevalence and diversity of constitutional mutations in the $p 53$ gene among $21 \mathrm{Li}-$ Fraumeni families. Cancer Res 54: 1298-1304

Chen J, Lindblom P and Lindblom A (1998) A study of the PTEN/MMAC1 gene in 136 breast cancer families. Hum Genet 102: 124-125

Foulkes WD, Flanders TY, Pollock PM and Hayward NK (1997) The CDKN2A (p16) gene and human cancer. Mol Med 3: 5-20

Hussussian CJ, Struewing JP, Goldstein AM, Higgins PAT, Ally DS, Sheahan MD, Clark WH Jr, Tucker MA and Dracopoli NC (1994) Germline p16 mutations in familial melanoma. Nature Genet 8: 15-21

Kamb A (1995) Cell-cycle regulators and cancer. TIG 11: 136-140

Li FP, Fraumeni JF Jr, Mulvihill JJ, Blattner WA, Dreyfus MG, Tucker MA and Miller RW (1988) A cancer family syndrome in twenty-four kindreds. Cancer Res 48: 5358-5362

Li J, Yen C, Liaw D, Podsypanina K, Bose S, Wang SI, Puc J, Miliaresis C, Rodgers L, McCombie R, Bigner SH, Giovanella BC, Ittmann M, Tycko B, Hibshoosh H, Wigler MH and Parsons R (1997) PTEN, a putative protein tyrosine phosphatase gene mutated in human brain, breast and prostate cancer. Science 275: $1943-1947$

Malkin D, Li FP, Strong LC, Fraumeni JF Jr, Nelson CE, Kim DH, Kassel J, Gryka MA, Bischoff FR, Tainsky MA and Friend SH (1990) Germ line p53 mutations in a familial syndrome of breast cancer, sarcomas, and other neoplasms. Science 250: 1233-1238

Myers MP, Stolarov JP, Eng C, Li J, Wang SI, Wigler MH, Parsons R and Tonks NK (1997) P-TEN, the tumor suppressor from human chromosome 10q23, is a dual-specificity phosphatase. Proc Natl Acad Sci USA 94: 9052-9057

Okamoto A, Demetrick DJ, Spillare EA, Hagiwara K, Perwez Hussain S, Bennett WP, Forrester K, Gerwin B, Serrano M, Beach DH and Harris CC (1994) Mutations and altered expression of $p 16^{1 N K 4}$ in human cancer. Proc Natl Acad Sci USA 91: 11045-11049

Quelle DE, Zindy F, Ashmun RA and Sherr CJ (1995) Alternative reading frames of the INK4a tumor suppressor gene encode two unrelated proteins capable of inducing cell cycle arrest. Cell 83: 993-1000

Steck PA, Pershouse MA, Jasser SA, Yung WKA, Lin H, Ligon AH, Langford LA, Baumgard ML, Hattier T, Davis T, Frye C, Hu R, Swedlund B, Teng DHF and Tavtigian SV (1997) Identification of a candidate tumour suppressor gene, $M M A C 1$, at chromosome 10q23.3 that is mutated in multiple advanced cancers. Nature Genet 15: 356-362

Tsou HC, Teng DH-F, Li Ping X, Brancolini V, Davis T, Hu R, Xun Xie X, Gruener AC, Schrager CA, Christiano AM, Eng C, Steck P, Ott J, Tavtigian SV and Peacocke M (1997) The role of MMAC1 mutations in early-onset breast cancer: causative in association with Cowden syndrome and excluded in BRCA1negative cases. Am J Hum Genet 61: 1036-1043

Varley JM, Evans DGR and Birch JM (1997a) Li-Fraumeni syndrome: a molecular and clinical review. Br J Cancer 76: 1-14

Varley JM, McGown G, Thorncroft M, Santibanez Koref MF, Kelsey AM, Tricker KJ, Evans DGR and Birch JM (1997b) Germline mutations of TP53 in Li-Fraumeni families: an extended study of 39 families. Cancer Res $\mathbf{5 7}$ : 3245-3252 